\title{
UKRAINIAN AND RUSSIAN BORROWINGS IN POLISH OF THE PETTY NOBILITY VILLAGE OF DOROHAN IN ZHYTOMYR REGION
}

\author{
O. V. ZAKHUTSKA, PhD: Doctor of Arts in Linguistics, Associate Professor, \\ National University of Life and Environmental Sciences of Ukraine
}

E-mail:oksana.zov@gmail.com

ORCID: 0000-0002-9766-0683

\begin{abstract}
The article presents Ukrainian and/or Russian borrowings used by Poles living in a small petty nobility village in Zhytomyr region in Ukraine in comparison with some other Polish dialects in the same territory. There are excerpted and analyzed 121 borrowed words divided into several thematic groups: "General notions" - e.g. budzień 'workday', nazad 'back', semia 'family'; "Person and his characteristics" - e.g. durny 'foolish, stupid, silly', harny 'nice, beautiful', Kacap 'of Russian nationality', pjanica 'drunkard'; "Activities and physical condition" - e.g. rozpowiadać 'to tell', wzuć się 'to put on one's shoes', otdychiwać 'to have a rest'; "Feelings, emotions and behaviour" - e.g. przezierać 'to despise, disdain', swarzyć się 'to argue, quarrel', wołnować się 'to worry about'; "Food, dishes and kitchenware" - e.g. hreczka 'buckwheat', deruny 'potato pancakes', kastrulka 'saucepan'; "State, administration and policy" - e.g. pensja 'retirement', partiejny 'belonging to a party', bryhadir 'brigadier'.

Among the borrowings words of Ukrainian origin prevail - there are 59 of them, i.e. about 49\%; there are 42 lexical units of possible Ukrainian and/or Russian origin, i.e. about 35\%, and 20 lexemes of strictly Russian origin, i.e. $16 \%$.

The comparison with other Polish dialects in Ukraine showed that out of 121 analyzed words 91 words are known and used also in other neighbouring settlements, what constitutes $75 \%$. The most similarities - 59 words, i.e. about $49 \%$, have been noted with the petty nobility dialect of Siaberka and peasant Oleshkivtsi and Hrechany.
\end{abstract}

Keywords: Polish in Ukraine, petty nobility, peasant, borrowing

Introduction. The Polish language, brought to Ukraine in the $16^{\text {th }}-19^{\text {th }}$ C. from ethnic Poland and preserved until now, is an important and unique part of the mutual Polish-Ukrainian cultural heritage. Before the World War II, Polish in Ukraine was spoken in various places. Currently, the situation is changing: compact Polish-speaking areas became less frequent and even relic, especially in the area left to the river Zbruch that belonged to the Russian Empire already since the end of the $18^{\text {th }} \mathrm{c}$.

Recent research and publications. The research conducted so far on the Polish language in Ukraine has shown that it reflects the local Poles' former state origin: peasant (chłopi) or petty nobility (szlachta). There have been published several monographic studies on phonetics and inflection system as well as on vocabulary of peasant dialects in Ukraine [cf. 5, p. 25-31], while the knowledge of Polish spoken by descendants of petty nobility is still scarce, limited to separate articles and one monograph on vocabulary of one dialect $[14$, p. 18-21]. Thus, it requires further theoretical and practical studying on a vast material basis from different places. 
Dorohan is a Polish petty nobility village in Zhytomyr region, $68 \mathrm{~km}$ to the west of Zhytomyr. The phonetic and inflectional features of its Polish language were generally presented by Yulia Yavorska as part of her research on the language of several Polish dialects in that territory and further described by Ewa Dzięgiel along with the history of the village, founded probably at the end of the $18^{\text {th }}$ - the beginning of the $19^{\text {th }} \mathrm{c}$. [3]. There was also analyzed one thematic layer of Dorohan vocabulary concerning food and culinary in comparison with the corresponding vocabulary of some other Polish dialects in Ukraine [13]. The necessity of considering vocabulary, both borrowed and native, from the point of view of its functioning and thematic variation was repeatedly pointed out by Janusz Rieger [7; 8]. The first steps in this direction were made by Aleksandra Krawczyk[Wieczorek] on the example of peasant dialect of Matskivtsi in Podilia [5; 9], Liudmyla Yanushevska and Oksana Zakhutska [4; 10; 12; 13], and Viktoriia Cherniak [2].

The purpose of this article is to present Polish vocabulary of Dorohan from the perspective of Ukrainian and Russian borrowings that inevitably appear in various thematic scopes in comparison with other Polish dialects in Ukraine. Such a description will become the next step in studying Polish vocabulary of descendants of petty nobility in Ukraine and will benefit to making its generalized vision in the future.

Data and methods. The material for this analysis was extracted from the texts recorded in Dorohan in 2002-2003 by A. Krawczyk[-Wieczorek], Marta Gugała and Genowefa Tymbrowska within the dialectical research conducted under the supervision of Prof. J. Rieger. This is a total of more than 10 hours of recordings (over 200 pages of typescript) from five respondents: KS22, MJ27, SB32, HR42, and JŚ49 (the digits indicate the year of birth).

The borrowings are presented thematically in comparison with corresponding vocabulary of neighbouring inhabitants of Siaberka (abbreviation: Siab) [11] and respondents of the petty nobility origin from Sataniv on the Zbruch River (abbreviation: Sat) [6] as well as with the vocabulary used in some peasant villages: in Oleshkivtsi and Hrechany based on the vocabulary by Iwona Cechosz-Felczyk (abbreviations:Oleshk, Hrech) [1], and in nearby Matskivtsi following A. Krawczyk[-Wieczorek] (abbreviation: Matsk) [5]. Within the defined thematic groups, words are given alphabetically, mostly in the basic form. Nouns, recorded only in plural, are given unchanged, similarly are described diminutives. Nouns (including diminutives) inevitably prevail, with a few adjectives and verbs.

Results. There have been excerpted 121 Ukrainian and/or Russian borrowings, including a few archaic, regional, and dialect Polish words that have parallels in contemporary Ukrainian and Russian (marked with an asterisk *). The lexemes are provisionally divided into several thematic groups; the division is dictated by the available material and some of its aspects can be developed and completed.

General notions [13]

budzień 'workday', Ukr./Rus. бlудень \ Siab; Oleshk

bukwa 'letter', Ukr./Rus. б/уква

Sat; Oleshk, Hrech, Matsk

chibla 'unless, except', Ukr. xiб/a

no record

chutko 'quickly, soon', Ukr. х'утко $\Delta$ no record 
kilko 'how many, a few', Ukr. dial. к'ілько А Siab; Oleshk, Hrech, Matsk

koncze 'for certain; very, extremely', Ukr. кlонче $\Delta$ no record

kudly 'where', Ukr. kydlu $\boldsymbol{\Delta}$ Siab

nazad" 'back', Ukr./Rus. наз/ad

Siab; Oleshk, Hrech, Matsk Siab

nikudy 'nowhere', Ukr. нікlyдu

odczynięty 'open', Ukr. відч/инений

$\Delta$ Oleshk

posydeńki 'get-together', Ukr. посид/еньки $\mathbf{\Delta}$ no record

semia 'family', Ukr. dial. с'ем'я (lit. $\operatorname{cim}^{\prime} / g$, cf. Rus. семь/я) \ Siab; Sat; Oleshk, Hrech, Matsk Hrech

tudly 'there', Ukr. mydlu A Siab;

Person and his characteristics [25]

chaziajka 'housewife; hostess', Ukr.

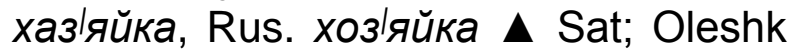
chadziajka, Hrech, Matsk

doglądzony 'taken care of; wellattended', cf. Ukr. догл/янутий \ no record

durny 'foolish, stupid, silly', Ukr.

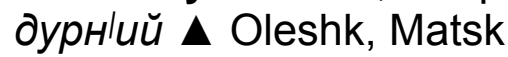

dziatki ${ }^{\star}$ dim. 'children', cf. Ukr. д/iтки, Rus. дlетки А Siab

gładki 'stout, fat', Ukr. гладк/uй Oleshk

harny, dim. harniuni 'nice, beautiful', Ukr. әlaрний, dim. dial. гарнюній $\mathbf{\Delta}$ Sat

Kacap 'of Russian nationality', Ukr. кац/ап $\boldsymbol{\Delta}$ no record

krepki 'sound, strong', Ukr. pot. кр/епкий, Rus. кр/епкий \ Oleshk, Hrech, Matsk

ludzina 'person', Ukr. люд/ина Siab; Oleshk, Hrech, Matsk

mołodycia, mołodyca 'young married woman', Ukr. молод/иця Oleshk, Hrech, Matsk

nierodny 'unrelated', Rus. неродн'ой $\boldsymbol{\Delta}$ Oleshk lopytny 'experienced', Rus. Іопытный $\Delta$ no record

pjanica 'drunkard', Rus. пь/яница А Siab; Oleshk, Hrech, Matsk

Polaczka 'Polish woman', Ukr./Rus. пол/ячка $\Delta$ Sat; Oleshk, Matsk

poprawić się 'to put on weight', Ukr. попр/авитися, Rus. попр/авиться А no record

posłuchniany 'obedient', Ukr. послухн/яний $\boldsymbol{\Delta}$ no record

przypytalski 'open-hearted, polite', cf. Ukr. прип/итливий $\Delta$ no record

pusty 'light-headed, thoughtless', Ukr. пуст/uй $\Delta$ no record

robotiaszczy 'hard-working', Ukr. робот/ящий, Rus. работ/ящий ৯ no record

sierjozny 'serious', Ukr. серйlозний, Rus. серь/ёзный $\boldsymbol{\Lambda}$ no record

splletnica 'gossip, newsmonger', Rus. спл/етница $\Delta$ Sat

splucha 'sleepyhead', Ukr. спл/юха $\Delta$ no record

uczony 'learned, erudite', Rus. уч/ёный $\Lambda$ Siab

wzrosły 'adult, grown-up', Rus. взрооллый А Siab; Oleshk, Hrech, Matsk

zamężny 'wealthy, affluent', Ukr. зам/ожний $\Delta$ Hrech zamożny

Activities and physical condition [12]

dawić się 'to choke', Ukr. дав/итися, Rus. дав/uться ৯ Siab; Hrech

nahodować dk 'to feed', Ukr. нагодув/amu \ Oleshk, Hrech

nakormić dk 'to feed', Ukr. накорм/umu $\mathbf{\Delta}$ no record

obidać ndk 'to have lunch/dinner', Ukr. обlidamu, cf. Rus. oбledamb $\boldsymbol{\Delta}$ no record

otdychiwać 'to have a rest', Rus. отдыхlamь, Ukr. pot. вiдduxlamu $\boldsymbol{\Delta}$ Sat

porozkidać 'to scatter, throw', Ukr. порозкид/amu $\mathbf{\Delta}$ no record 
rozpluszczyć 'to open one's eyes', Ukr. розпл/ющити $\boldsymbol{\Delta}$ no record

rozpowiadać 'to tell', Ukr. розповід/amu \ Siab; Sat; Oleshk, Hrech

śniadać ${ }^{\star}$ dk / pośniadać dk 'to have breakfast', Ukr. $\mathrm{CH}^{\prime}$ idamu Oleshk, Hrech

spożywać 'to eat, consume', Ukr. споживlamu ৯ Siab; Sat

wieczerzać * 'to have supper', Ukr. веч/еряти \ Oleshk, Hrech

wzuć się 'to put on one's shoes', Ukr. вз/ymucя $\boldsymbol{\Delta}$ Oleshk

Feelings, emotions and behavior [13]

bażać 'to want, wish', Ukr. бажlamu \ Siab; Oleshk, Hrech, Matsk

drużyć 'to be friends', Ukr. друж/uти, Rus. друж/uть А no record

myrzyć się 'to reconcile; to leave peacefully', Ukr. мир/uтися, Rus. мир'uться \ Siab mirzyć się

naśmiechać się 'to mock', Ukr. насміхатися, Rus. насмехаться no record

niedowolny

'dissatisfied, displeased', Ukr. pot. недов/ольний, Rus. недовольный $\boldsymbol{\Delta}$ no record

przezierać 'to despise, hold in contempt; disdain', Rus. презир/amь Sat Matsk

spletni 'rumours', Rus. сплlетни

swarzyć się to argue, quarrel', Ukr. свар/umucя \ Siab; Oleshk, Matsk

turbować się 'to worry about, feel disturbed', Ukr. турбувlaтися Oleshk

uważać 'to respect, honour', Rus. уважать \ Sat; Oleshk

wołnować się 'to worry about, feel disturbed', Rus. волнов/аться \ Siab (cf. Hrech wołnienie)

zabocić się 'to take care of, look after', Rus. заб/omumьcя $\boldsymbol{\Delta}$ no record znuszczać się 'to mock at; humiliate', Ukr. знущ'атися \ Oleshk, Hrech

Food, dishes and kitchenware [34]

charcze pl 'food', Ukr. sg харч, pl xapuli $\Delta$ Siab

chlibyna 'a loaf of bread', Ukr. хліб/ина $\boldsymbol{\Delta}$ no record

chłódek 'jellied minced meat', cf. Ukr. холод/ець, Rus. холод/ец \ Siab (cf. Sat Ukr. chołodec, chołodilec, studzień, Matsk Ukr. dial. drahla)

czaj 'tea', Ukr./Rus. чай \ Siab; Oleshk, Hrech, Matsk

czerpaczek dim. 'ladle', Ukr./Rus. dim. черпачlок $\boldsymbol{\Delta}$ Siab; Oleshk, Hrech

deruny 'potato pancakes', Ukr. Im дерун'и (Ip дер/ун) $\boldsymbol{\Delta}$ Siab; Hrech

hreczanny 'buckwheat', Ukr. греч/аний $\Delta$ Siab; Sat; Oleshk, Hrech

hreczka 'buckwheat', Ukr. гр/ечка \ Siab; Sat; Oleshk, Hrech

kartofla 'potatoes', Ukr. dial. карт/офрля А Siab; Sat; Oleshk, Hrech

kartoszka 'potatoes', Rus. картоошка \ Hrech

kastrulka dim. 'saucepan', Ukr. dim. кастр/улька \ Siab; Sat; Oleshk, Hrech, Matsk

kisły 'sour milk', Ukr. к'ислий, Rus. к'ислый А Siab; Hrech

kopczony 'bloated', Ukr. к'опчlений, Rus. копчё̈нный \ Siab; Hrech Rus. kapczony

krużka 'cup', Rus. кр/ужка А Hrech

kukuruza 'maize, corn', Rus. кукур/уза А Siab; Sat; Oleshk, Hrech, Matsk

mysnik 'dresser', Ukr. м'исник А Siab pampuszki pl 'pancakes', Ukr. пампушк'u, Rus. памп'ушки А Siab; Oleshk

pasznia 'crop', Ukr. пашнlя \ Siab

płyta 'kitchen range, cooker', Ukr. плитla \ Siab; Oleshk, Matsk 
płytka 'electric stove', Ukr. пл/итка - Siab; Oleshk posuda 'tableware, dishes', Rus. посlyda, cf. Ukr. пlocyd Siab; Oleshk, Hrech, Matsk

pszono 'millet meal', Ukr. пшон'о

Siab; Oleshk, Hrech

rys 'rice', Ukr. puc \ Siab; Hrech, Matsk

skibka 'a piece of bread', Ukr. ск'ибка \ Siab; Hrech

sup 'soup', Ukr./Rus. суп $\boldsymbol{\Delta}$ Oleshk, Hrech, Matsk

śniadanek 'breakfast', Ukr. снід/анок \ Siab; Oleshk, Hrech (along with śniadanie)

tariłka 'plate', Ukr. тар/ілка Oleshk Rus. tarełka

tuchły 'rotten, bad', Ukr. туухлий, Rus. mуухлый $\mathbf{\Delta}$ Siab; Hrech

tuszonka 'tinned stewed meat', Ukr. тушіонка, Rus. тушіёнка \ Siab; Oleshk, Hrech, Matsk

uksus 'vinegar', Rus. 'уксус Hrech

wermyszel 'spaghetti', Ukr. вермишіель А Hrech Rus. wiermiszel

wkusny 'delicious, tasty', Rus. вк'усный $\boldsymbol{\Delta}$ Hrech, Matsk

żyr 'fat', Ukr./Rus. жup \ Siab; Sat; Oleshk, Hrech, Matsk

żyrny 'fatty', Ukr. ж/ирний, Rus. ж/ирный \ Siab; Sat; Oleshk, Hrech

State, administration and policy [24]

batkiwszczyna 'fatherland', Ukr. батьківщ/ина ৯ Oleshk, Hrech

brygadirowa 'here: brigadier's wife', Ukr./Rus. бригаd/upова $\Delta$ no record

bryhadir 'brigadier', Ukr./Rus. бpuzadlup \ Oleshk, Hrech, Matsk

głodówka, hołodowka 'famine of 1946', Ukr./Rus. голод/овка \ Oleshk, Hrech, Matsk

kolektyw 'collective farm', Ukr. колект/uв \ Siab; Oleshk, Hrech

kothosp 'collective farm', Ukr. колг/осп \ Sat; Oleshk, Hrech, Matsk kooperacja 'village shop', Ukr. dial. кооперlація $\boldsymbol{\Delta}$ no record

manaszka, monaszka 'nun', Rus. мон/ашка \ Matsk

maszyna 'car', Ukr./Rus. маш'ина

\ Siab; Oleshk, Hrech, Matsk

matuszka 'priest's wife', Ukr./Rus. матушка $\Delta$ no record

modistka 'milliner, modiste', Rus. мод/истка $\Delta$ no record

partiejny '(belonging to) party', Ukr. парт/ійний, Rus. парт/ийный $\boldsymbol{\Delta}$ no record

pasport 'passport, ID card', Ukr.I Rus. пlacпорm \ Siab; Sat; Oleshk, Matsk

patron 'cartridge', Ukr./Rus. патр/он $\boldsymbol{\Delta}$ no record

pensja 'pension', Ukr. пеенсія Siab; Sat; Oleshk, Hrech, Matsk

razwiedka 'reconnaissance', Rus. разведка $\boldsymbol{\Delta}$ no record

represirować 'to subject to repression', Ukr. репресувlamu, Rus. репресcluровать $\boldsymbol{\Delta}$ Siab; Matsk

sawiecka włast' 'Soviet government', Rus. сов/етская власть \ Siab; Sat; Oleshk, Hrech, Matsk

silrada 'rural council', Ukr. сільськla plada \ Siab; Sat; Oleshk, Hrech, Matsk sołdat 'soldier', Ukr./Rus. солд/am \ Siab; Sat; Oleshk, Hrech

stancija 'station', Ukr. станиія, Rus. станция \ Siab; Sat; Oleshk, Hrech, Matsk

tiurma 'prison', Ukr. тюрмla, Rus. тюрьм'а \ Siab; Sat; Oleshk

towarniak 'goods wagon', Ukr./Rus. товарн/як \ Oleshk, Hrech, Matsk

zbory 'meeting, gathering', Ukr. збlори \ Siab; Oleshk, HrechThe analyzed lexical borrowings (121 units) are presented thematically. "General notions" group is not numerous (13); borrowings here denote time, space and direction - e.g. budzień, kudly, nikudy, 
nazad, as well as words like semia and posydeńki.

"Person and his characteristics" group is twice bigger (25); it includes borrowings connected with people and their personalities - e.g. chaziajka, ludzina, mołodycia, uczony, as well as characteristics based on one's nationality - e.g. Kacap, Polaczka, appearance - e.g. gładki, krepki, harny, doglądzony, traits of character - e.g. durny, przypytalski, sierjozny, pusty, and habits - e.g. pjanica, spletnica, splucha, etc. Here nouns and adjectives prevail (9 and 15 words) with only one verb poprawić się noted.

"Activities and physical condition" group (12) presents a few borrowings naming people's everyday actions - e.g. rozpowiadać, spożywać, wzuć się, and physical condition - e.g. dawić się, otdychiwać, etc. All of them are verbs.

"Feelings, emotions and behaviour" group (13) contains mostly verbs, but for one noun and one adjective, denoting a person's emotional state and behavior e.g. bażać, drużyć, myrzyć się, przezierać, swarzyć się, wołnować się, niedowolny, spletni, etc.

"Food, dishes and kitchenware" group is the most numerous one (34); includes borrowings denoting food and food products - e.g. hreczka, kartofla, kukuruza, pasznia, dishes and their ingredients - e.g. chłódek, deruny, pampuszki, tuszonka, as well as names of kitchenware used in people's everyday life - e.g. czerpaczek, kastrulka, krużka, mysnik.

"State, administration and policy" group (24) presents borrowings related to authorities and their activities, including historic ones - e.g. pensja, pasport, kothosp, kolektyw, partiejny, sawiecka włast', zbory; also names of professions and military notions can be found here - e.g. bryhadir, modistka, sołdat, patron.

Among the borrowings words of Ukrainian origin prevail - there are 59 of them, i.e. about $49 \%$; there are 42 lexical units of possible Ukrainian and/or Russian origin, i.e. about $35 \%$, and 20 lexemes of strictly Russian origin, i.e. $16 \%$.

The comparison with other Polish dialects in Ukraine showed that out of 121 Ukrainian and/or Russian borrowings noted in Dorohan 91 words appear also in other Polish places in the same territory, what constitutes $75 \%$. The most parallels have been found with the neighbouring petty nobility Siaberka - 59 words and peasant Oleshkivtsi and Hrechany -59 and 58 words, i.e. about $49 \%$ correspondingly. Fewer similarities have been noted between respondents from the petty nobility from Sataniv -27 words, i.e. $22 \%$, and peasant Matskivtsi - 38 words, i.e. $31 \%$. The existence of "newer" borrowings in the vocabulary of Dorohan may be explained by their latest provenance and growing Ukrainian and/or Russian influences.

Conclusions and discussion. The article presents a not described before borrowed vocabulary of the petty nobility dialect of Dorohan in comparison with other Polish dialects in Ukraine. The analyzed linguistic material can become the basis for further cultural, ethnographic or anthropological research in the conditions of irreversible disappearance of Polish in the former South-Eastern Poland.

\section{References}

1. Cechosz-Felczyk, I. (2004). Słownictwo gwary Oleszkowiec i Hreczan (Greczan) na Podolu [Vocabulary of Oleshkivtsi and Hrechany (Grechany) dialects in Podilia]. Kraków: Lexis, 397.

2. Cherniak, V. (2017). Nazwy odzieży w Wójtowcach na Podolu na tle określeń w wybranych gwarach polskich

\section{(c) O. V. Zakhutska}

«International journal of philology» | «Міжнародний філологічний часопис» Vol. 10, № 1, 2019 
na Ukrainie [Garment names in Wojtowce in Podolia in comparison with other Polish dialects in Ukraine]. Slavia Orientalis, Vol. LXVI, No. 4, 687-708.

3. Dzięgiel, E. (2007). Dorohań [Dorohan]. J. Rieger, I. Cechosz-Felczyk, E. Dzięgiel, Język polski na Ukrainie w końcu XX wieku [The Polish language in Ukraine at the end of the 20th c.]. Cz. 2. Polszczyzna w Lwowskiem, Żytomierskiem i na Podolu. Teksty. Kraków: Lexis, 223-234.

4. Januszewska, L., Zakhutska, O. (2012). Obrzędowa leksyka weselna wybranych wsi szlacheckich i chłopskich za Zbruczem (na przykładzie wsi Siaberka i Hreczany) [Wedding vocabulary of chosen noble and peasant villages to the left of the Zbruch river (on the example of Siaberka and Hrechany villages)]. Język polski dawnych Kresów Wschodnich. Polskie dziedzictwo językowe na dawnych Kresach [The Polish language of the former Eastern Borderlands. Polish linguistic heritage in the former Borderlands]. Vol. 5, ed. Ewa Dzięgiel, Katarzyna Czarnecka and Dorota A. Kowalska. Warsaw: Wydawnictwo Naukowe Semper, 93-101.

5. Krawczyk, A. (2007). Zapożyczenia leksykalne w sytuacji wielojęzyczności. Ukrainizmy i rusycyzmy w gwarze Maćkowiec na Podolu [Lexical borrowings in the situation of multilingualism. Ukrainian and Russian borrowings in Matskivtsi dialect in Podilia]. Warsaw: DiG, 157.

6. Modzelewska, R., Rieger, J. (2008). Słownictwo gwary Satanowa nad Zbruczem [Vocabulary of Sataniv dialect at the Zbruch river]. Słownictwo kresowe. Studia i materiały, ed. J. Rieger, Warsaw, 270-366.

7. Rieger, J. (2004). Jak badać współczesne słownictwo kresowe? [How to study modern Polish vocabulary in the former Eastern Borderlands?]. Studia nad polszczyzną kresową, Vol. XI, ed. J. Rieger. Warsaw: Wydawnictwo Naukowe Semper, 23-33.

8. Rieger, J. (2012). O słownictwie dzisiejszej polszczyzny na Ukrainie i metodach jej badania [On vocabulary of modern Polish in Ukraine and methods of its studying]. Prilozi na Oddelenieto za lingvistika i literaturna nauka pri MANU", br. 37///2011, ed. M. Mirkulovska. Skopje, 121-128.

9. Wieczorek, A. (2011). Słownictwo polskiej gwary kresowej na przykładzie Maćkowiec na Podolu. Charakterystyka funkcjonalna [The vocabulary of the Polish dialect on the example of Matskivtsi in Podolia. Functional characteristics]. Warsaw, 314. [doctoral thesis]

10. Zakhutska, O. (2011). Jeszcze o słownictwie kresowym (próba porównania idiolektów szlacheckich i chłopskich na Podolu) [Once again on vocabulary in the former Eastern Borderlands (an attempt to compare noble and peasant idioletes in Podolia)]. Język polski, XCI, 5, 352-363.

11. Zakhutska, O. (2015). Polszczyzna drobnoszlacheckiej wsi Siaberka na Wołyniu. Słownictwo [The Polish language of petty nobility village of Siaberka in Volhynia. Vocabulary]. Warsaw: Wydawnictwo DiG, 200.

12. Zakhutska, O. (2017). Names of family relations in petty nobility village of Siaberka in Zhytomyr region. Naukovyi visnyk Natsionalnoho universytetu bioresursiv i pryrodokorystuvannia Ukrainy. Seriia : Filolohichni nauky, Vyp. 272, 23-29.

13. Zakhutska, O. (2018). Słownictwo kulinarne gwary polskiej wsi Dorohań w Żytomierskiem [Culinary vocabulary of the Polish dialect of Dorohan in Zhytomyr region]. Slavia Orientalis, Vol. LXVII, No. 2, 325-349. 


\section{УКРАЇНСЬКІ ТА РОСІЙСЬКІ ЗАПОЗИЧЕННЯ У ПОЛЬСЬКІЙ МОВІ ДРІБНОШЛЯХЕТСЬКОГО СЕЛА ДОРОГАНЬ НА ЖИТОМИРЩИНІ \\ О. В. Захуцька}

Анотація. У статті представлено українські та/або російські запозичення, вживані у польській мові мешканців невеликого дрібношляхетського села Дорогань у Житомирській області, у порівнянні з лексикою деяких сусідніх польських говірок в Україні. Проаналізовано 121 запозичення, які поділено на кілька тематичних груп: «Загальні поняття» - напр. budzień $\diamond$ укр. будень, nazad $\diamond$ укр. наз ад, semia $\diamond$ укр. сім'я; «Людина та їі характеристики» - напр. durny $\diamond$ укр. дурн'ий, harny $\diamond$ укр. гарний, Касар $\diamond$ укр. кацап, pjanica $\diamond$ укр. п'яниця, рос. пьяница; «Дії та фрізичний стан» - напр. rozpowiadać $\diamond$ укр. розповідати, wzuć się $\diamond$ укр. вз утися, otdychiwać $\diamond$ рос. отдыхать, укр. розм. отдихати; «Почуття, емоції та поведінка» - напр. przezierać $\diamond$ рос. презирать, swarzyć się $\diamond$ укр. свар итися, wołnować się $\diamond$ рос. волноваться; «їжа, продукти харчування та посуд» - напр. hreczka $\diamond$ укр. гречка, deruny $\diamond$ укр. дерун'и, kastrulka $\diamond$ укр. каструлька; «Держава, адміністрація та політика» - напр. pensja $\diamond$ укр. пенсія, partiejny $\diamond$ укр. партійний, poc. партийный, bryhadir $\diamond$ укр./poc. бригад'ир.

Серед запозичень переважають українізми - 59 лексичних одиниць, тобто близько 49 \%; 42 лексичні одиниці, тобто близько 35 \%, можуть походити як 3 української, так і з російської мов; 20 лексичних одиниць, тобто $16 \%$, запозичено з російської мови.

Порівняння з іншими польськими діалектами в Україні показало, що з 121 запозичення 91 слово використовується також в інших сусідніх територіях, що становить 75\%. Найбільше подібностей - 59 слів, тобто близько 49\%, було відзначено з сусіднім дрібношляхетським селом Сяберка та холопськими селами Олешківці і Гречани, що знаходяться поблизу Хмельницького.

Ключові слова: польська мова в Україні, дрібна шляхта, холоп («селянин»), запозичення

(C) O. V. Zakhutska

«International journal of philology» | «Міжнародний філологічний часопис» Vol. 10, № 1, 2019 